\title{
Chromosomal Alterations in Patients with Alzheimer Disease in Manaus, Amazonas, Brazil
}

Cleiton Fantin ${ }^{1}$, Kledson Moraes Nunes ${ }^{1}$, Ernanda Raquel de Queirós Gonçalves de Sousa e Fernandes ${ }^{2,3}$, Diana Vieira Brito ${ }^{1}$, Natalia Dayane Moura Carvalho ${ }^{1}$ and Denise Corrêa Benzaquem ${ }^{1}$

1. Laboratório de Citogenética, Escola Superior de Ciências da Saúde, Universidade do Estado do Amazonas, Manaus, Amazonas 69065-001, Brazil

2. Laboratório de Análises Clínicas do Hospital Universitário Getúlio Vargas-HUGV, Manaus, Amazonas 69020-170, Brasil

3. Secretaria Municipal de Saúde, SEMSA, Manaus, Amazonas 69057-000, Brasil

\begin{abstract}
Alzheimer disease (AD) is a complex neurodegenerative pathology that is characterized by a cognitive decline. Its causes and mechanisms are still largely unknown. It has been suggested that both genetic and life exposure factors can contribute to AD development. There are also evidences that chromosomal alterations can be related to this disease. So far, there is not a precise diagnosis for $\mathrm{AD}$, which is given only after the exclusion of other dementia by clinical and neurological examination. The possible association of $\mathrm{AD}$ with chromosomal alterations and the easy access of classical cytogenetics analysis are important aspects to consider, given the difficulties in diagnosis. Due to the lack of similar studies in Brazil and the increasing number of AD cases in the state of Amazonas, the aim of this study was to investigate the presence of chromosomal alterations in patients diagnosed with $\mathrm{AD}$ in Manaus, Amazonas, Brazil. Peripheral blood lymphocytes of twelve patients and twelve healthy individuals with the same age were analyzed using conventional karyotyping. All AD patients presented cells with autosomal aneuploidy, while no chromosomal alterations were found in the age-matched controls. Also, rare events of double and multiple aneuploidies are being reported in association with AD for the first time. Our results corroborate that the increase in the frequencies of aneuploidies is not related to the aging process itself, but it might be associated to the disease development. However, no chromosomes were preferentially affected in all AD patients, and no consistent karyotype pattern for AD lymphocytes was found. Therefore, our results do not support the use of standard cytogenetics as a tool for AD diagnosis. Future studies are necessary to understand better the association between chromosomal alterations and AD.
\end{abstract}

Key words: AD, karyotyping, numeric chromosomal alterations, double aneuploidies, multiple aneuploidies.

\section{Introduction}

Alzheimer disease (AD) is a complex neurodegenerative pathology that is characterized by a cognitive decline, with neuropsychological symptoms such as amnesia, deficits in language abilities and executive dysfunction [1]. There are two major AD types: early onset or familial, the rarer form, which occurs in people roughly from 30 to 65 years old, and it is usually inherited; and late-onset or sporadic, the most common form, which affects people over 65 years old [2, 3]. There are two neuropathological features that have been considered as the hallmarks of this

Corresponding author: Cleiton Fantin, Ph.D., professor, research fields: genetics and cytogenetics. disease, which are the extracellular plaques formed by beta-amyloid peptides, and the intracellular tangles of hyperphosphorylated microtubule-associated protein tau (MAPT) [4]. However, these features can only be confirmed postmortem. Thus, the diagnosis of probable $\mathrm{AD}$ is made only by clinical and neurological examination after the exclusion of other neurodegenerative disorders [5].

Several studies have been made in order to elucidate the causes and mechanisms of $\mathrm{AD}$. It has been suggested that genetic variants and life exposure factors, such as obesity, diabetes, cardiovascular diseases, infectious agents and chronic inflammations can contribute to $\mathrm{AD}$ development [2, 4, 6, 7]. Genetic risk factors are considered preponderant in $\mathrm{AD}$ 
etiology. At first, genetic linkage and positional cloning studies lead to the discovery of three genes that encode proteins involved in beta-amyloid production: the amyloid precursor protein (APP) and the presenilins (PSEN1 and PSEN2). Autosomal dominant inheritance of early-onset $\mathrm{AD}$ is linked to highly penetrant mutations in these genes [2, 5]. However, variations in these genes can explain only a small percentage of cases, even in familial AD [4]. For late-onset $\mathrm{AD}$, the apolipoprotein $\mathrm{E}$ (APOE) gene was the first one that was associated with an increased risk of the disease [3]. More recently, genome-wide association studies were able to identify eleven risk genes, which are involved in pathways such as lipid metabolism, immune system and synaptic functioning mechanisms [3, 4].

Besides the risk genes, there are evidences that chromosomal alterations can play a role in $A D$ development. Cytogenetic studies performed in $\mathrm{AD}$ patients revealed an increased occurrence of aneuploidies involving sexual (X chromosome) and autosomal chromosomes in different cell types [8-15]. Specific types of aneuploidies have also been reported in $\mathrm{AD}$ patients, especially for chromosome 21 [16]. Due to neuropathologic parallels between $\mathrm{AD}$ and Down syndrome (DS), it has been proposed that neuronal trisomy 21 mosaicism could be a mechanism of $\mathrm{AD}$ pathogenesis $[17,18]$. These reports of aneuploidies associated with $\mathrm{AD}$ raised the possibility of using karyotype analysis as a tool for helping diagnose [19]. However, there have been controversies over this issue. Some authors contested the strength of that association and stated that the observed aneuploidies could be related to other factors, such as aging $[8,9]$. Furthermore, the link between chromosome alterations and AD pathogenesis is still not well defined [17].

Therefore, the aim of this study was to analyze cytogenetically patients diagnosed with AD in Manaus, Amazonas, Brazil. We investigated the presence of chromosomal alterations in peripheral blood lymphocytes of $\mathrm{AD}$ patients and compared with age-matched controls. Moreover, we discussed if aneuploidies could be associated with disease traits observed in the patients, and if there was a consistent pattern of chromosomal alterations detected in $\mathrm{AD}$ patients that could be used to help diagnose $\mathrm{AD}$ in the hospitals of Manaus.

\section{Material and Methods}

This study was approved by the Research Ethics Committee of the Amazonas State University under authorization number 2.456.885 (CAAE: 66630616.6.0000.5016). Patients were indicated by Dr. Talísia Vianez from the Department of Neurology at the University Hospital Getúlio Vargas and the Outpatient Clinic Araújo Lima. Twelve individuals (four men and eight women) clinically diagnosed with $\mathrm{AD}$ for at least three years were chosen for this study. Twelve healthy individuals of the same age range were selected as the control group. The research purpose was explained to all participants and they voluntarily agreed to sign an informed consent form, as required by the National Health Council. AD patients were submitted to the mini-mental state examination (MMSE) to evaluate their cognitive function [20]. MMSE scores were used to classify the cognitive impairment of the patients in three levels: $24-30=$ no cognitive impairment; 18-23 = mild cognitive impairment; and 0-17 = severe cognitive impairment [21].

Cytogenetic analyses were carried out at the Laboratory of Cytogenetics of the Amazonas State University. Metaphase chromosomes were obtained from the culture of peripheral blood lymphocytes [22]. G-banding was performed using trypsin solution [23]. The best metaphases were selected and photographed using the optical microscope Nikon Eclipse 2000 with a field camera, light field, and a 100× objective lens. One hundred cells were analyzed for each patient and control subject. Metaphases were karyotyped using the GeneAll software. Chromosomal alterations were 
classified according to the international system for human cytogenetic nomenclature (ISCN).

\section{Results}

A brief clinical description of the $\mathrm{AD}$ patients analyzed in this study is provided in Table 1 . They were aged between 62-85 years and had been diagnosed with the disease for 3-7 years. According to their scores in MMSE, ten patients were in the category of severe cognitive impairment (0-15 points), among which four did not score any point; and two patients were in the category of mild cognitive impairment (18-21 points).

We analyzed one hundred metaphase cells of each individual. All $\mathrm{AD}$ patients presented cells with numeric chromosomal alterations, which can be classified as autosomal aneuploidy, with loss of one chromosome, found in all twelve patients (Fig. 1a); double aneuploidy, with loss of one sexual and one autosomal chromosome or two autosomal chromosomes, found in five patients (Fig. 1b); and multiple aneuploidy, with the loss of three autosomal chromosomes, found in one patient (Fig. 1c). These results are summarized in Table 2. No chromosomal alterations were observed in the karyotypes of individuals from the control group.

\section{Discussion}

$\mathrm{AD}$ is a complex brain disorder and many environmental, genetic or epigenetic factors can be associated with its development [24]. Cytogenetic analyses have demonstrated that chromosomal instability events, including aneuploidy, could be one of these factors [10, 25].

The occurrence of aneuploidies in $\mathrm{AD}$ patients has been investigated for different cell types. An increase in aneuploidy (loss and gain) for distinct chromosomes was reported in lymphocytes of AD patients [10, 26, 27]. Aneuploidy of specific chromosomes was also reported in AD lymphocytes [24]. Moreover, high levels of aneuploidy were observed also in fibroblasts and buccal cells of AD patients [28, 29]. Also, Iourov et al. [16] found a significant increase of monosomy and trisomy for chromosome 21 in brain cells of AD patients. Interestingly, there is a parallel between DS and $\mathrm{AD}$, since $\mathrm{DS}$ patients over 40 years old develop a brain pathology indistinguishable from AD [30]. It has been proposed that aneuploidy of chromosome 21 could be one of the mechanisms of $\mathrm{AD}$ neuropathogenesis [18, 30, 31].

Evidence of the occurrence of aneuploidies in distinct cell types suggested that there is a systemic chromosome instability in AD patients. This led to the

Table 1 Description of the AD patients chosen for this study: age, time since diagnosis and MMSE scores are shown. The symbol “_” was used for unavailable data.

\begin{tabular}{llll}
\hline Patient & Age (years) & Diagnosis time (years) & MMSE scores \\
\hline C-01 & 71 & 6 & 6 \\
C-02 & 79 & 4 & 0 \\
C-03 & 79 & 7 & 15 \\
C-04 & 79 & - & 0 \\
C-05 & 86 & 5 & 8 \\
C-06 & 81 & - & 9 \\
C-07 & 79 & 4 & 5 \\
C-08 & 62 & - & 21 \\
C-09 & 74 & 5 & 12 \\
C-10 & 81 & 3 & 0 \\
C-11 & 77 & 3 & 0 \\
C-12 & 85 & - & 18 \\
\hline
\end{tabular}




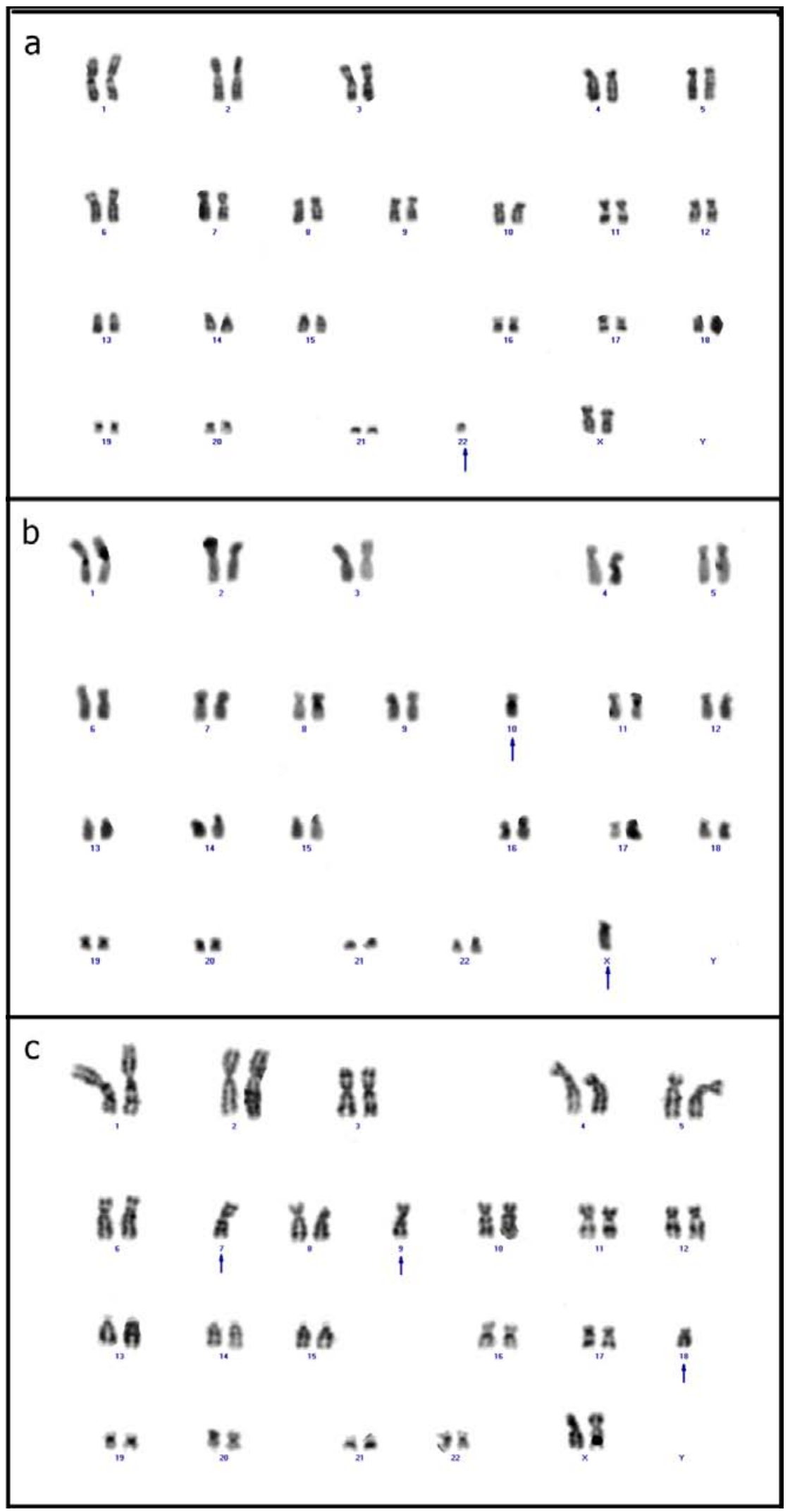

Fig. 1 Karyotypes of patients diagnosed with Alzheimer disease: (a) loss of one homolog of the chromosomal pair 22; (b) loss of one homolog of the chromosomal pair 22 and the sexual chromosome X; (c) loss of one homolog of the chromosomal pairs 7 , 9 and 18. 
Table 2 Chromosomal alterations found in peripheral blood cells of the AD patients analyzed in this study. $N=$ number of cells found in the total of $\mathbf{1 0 0}$ analyzed cells. The symbol “_” was used for unavailable data.

\begin{tabular}{|c|c|c|c|c|}
\hline \multirow{2}{*}{ Patient } & \multicolumn{3}{|c|}{ Aneuploidy } & \multirow[b]{2}{*}{$N$} \\
\hline & Aneuploidy of autosomal chromosomes & Double aneuploidies & Multiple aneuploidies & \\
\hline \multirow{4}{*}{ C-01 } & $45, \mathrm{XX},-6$ & - & - & 1 \\
\hline & $45, X X,-9$ & - & - & 1 \\
\hline & $45, X X,-18$ & - & - & 1 \\
\hline & $45, X X,-22$ & - & - & 1 \\
\hline \multirow{2}{*}{ C-02 } & $45, X Y,-18$ & - & $\bar{c}$ & 1 \\
\hline & $45, X Y,-19$ & - & - & 1 \\
\hline \multirow{3}{*}{ C-03 } & $45, X Y,-5$ & - & - & 1 \\
\hline & $45, X Y,-17$ & - & - & 2 \\
\hline & $45, X Y,-18$ & - & - & 2 \\
\hline \multirow{3}{*}{ C-04 } & $45, X X,-9$ & - & - & 1 \\
\hline & $45, X X,-18$ & - & - & 1 \\
\hline & $45, X X,-22$ & - & - & 1 \\
\hline \multirow{3}{*}{ C-05 } & $45, X X,-9$ & - & - & 1 \\
\hline & $45, X X,-18$ & - & - & 1 \\
\hline & - & $44, X,-X,-10$ & - & 1 \\
\hline \multirow{5}{*}{ C-06 } & $45, X Y,-9$ & - & - & 2 \\
\hline & $45, X Y,-12$ & - & - & 1 \\
\hline & $45, X Y,-18$ & - & - & 1 \\
\hline & $45, X Y,-21$ & - & - & 1 \\
\hline & - & $44, X Y,-3,-18$ & & 1 \\
\hline \multirow{6}{*}{ C-07 } & $45, X X,-9$ & - & - & 3 \\
\hline & $45, X X,-12$ & - & - & 2 \\
\hline & $45, X X,-13$ & - & - & 1 \\
\hline & $45, X X,-15$ & - & - & 2 \\
\hline & $45, X X,-21$ & - & - & 1 \\
\hline & - & $44, X,-X,-21$ & - & 1 \\
\hline \multirow{4}{*}{ C-08 } & $45, X X,-4$ & - & - & 1 \\
\hline & $45, X X,-19$ & - & - & 1 \\
\hline & - & $44, X,-X,-13$ & - & 1 \\
\hline & - & - & $43, X X,-7,-9,-18$ & 1 \\
\hline \multirow{4}{*}{ C-09 } & $45, X X,-19$ & - & - & 2 \\
\hline & $45, X X,-21$ & - & - & 1 \\
\hline & $45, X X,-22$ & - & - & 1 \\
\hline & - & $44, X X,-16,-20$ & - & 1 \\
\hline \multirow{3}{*}{$\mathrm{C}-10$} & $45, X X,-8$ & - & - & 1 \\
\hline & $45, X X,-18$ & - & - & 2 \\
\hline & $45, X X,-20$ & - & - & 1 \\
\hline $\mathrm{C}-11$ & $45, X X,-14$ & - & - & 1 \\
\hline \multirow{2}{*}{ C-12 } & $45, X Y,-13$ & - & - & 1 \\
\hline & $45, X Y,-21$ & - & - & 1 \\
\hline
\end{tabular}

proposal that aneuploidy frequency could be used as an $\mathrm{AD}$ biomarker and could contribute to the diagnosis of this complex disease [19, 27, 32]. However, there has been a debate about whether aneuploidies are associated with $\mathrm{AD}$ pathogenesis itself or if they are a consequence of other related factors. Some authors suggested that the increased aneuploidy in AD patients is a consequence of normal aging [8, 9, 33]. This is supported by the fact that loss of sexual and autosomal chromosomes is common in the cellular aging process, and $\mathrm{AD}$ is considered a systematic disease that accelerates aging [34].

Here we found that all AD patients presented peripheral blood lymphocytes with aneuploidies, while no chromosomal losses or gains were observed in healthy individuals within the same age range. More remarkably, double and multiple aneuploidies are being reported for the first time in AD patients. Three double aneuploidy cases involved the loss of one of the sexual chromosomes (X chromosome) and autosomal 
chromosomes (one homolog of pairs 10, 13 and 21), while two cases involved the loss of two autosomal chromosomes (one homolog of pairs 3, 16, 18 and 20). The case of multiple aneuploidies involved the loss of three autosomal chromosomes (one homolog of pairs 7 , 9 and 18). The occurrence of double and multiple aneuploidies in humans is a relatively rare event. Most cases reported are sexual chromosomal aneuploidies combined with trisomy 21 [35, 36]. The mechanism that originates these types of aneuploidies is unclear. It has been hypothesized that double and multiple aneuploidy results either from two or more nondisjunction events in gametogenesis or from a single nondisjunction event in a trisomic zygote [37]. Therefore, our results corroborate that aneuploidies are associated to $\mathrm{AD}$ development.

Aneuploidy observed in the patients studied here involved the loss of different chromosomes (one homolog of pairs 3, 4, 5, 6, 7, 8, 9, 10, 12, 13, 14, 15, 16, 17, 18, 19, 20, 21 and 22). At the intraindividual level, preferential chromosome loss was not observed. At the interindividual level, the loss of some chromosomes was observed in a higher frequency: 18 (eight patients), 9 (six patients), 21 (four patients), 13 (three patients) and 19 (three patients). Migliore et al. [25] observed a particularly higher frequency in aneuploidy of chromosomes 13 and 21 in lymphocytes of $\mathrm{AD}$ patients. Also, Geller and Potter [28] reported an increased aneuploidy of chromosomes 18 and 21 in fibroblasts, and Thomas and Fenech [29], a higher aneuploidy of chromosomes 17 and 21 in buccal cells of AD patients. In this study, however, there was no preferentially affected chromosome in all cases. Also, we could not detect a consistent chromosomal pattern that could be used as indicative of an AD-specific type of aneuploidy. Thus, even though we found a strong association of aneuploidies with $\mathrm{AD}$, our results do not support the use of standard cytogenetic analysis as a way to contribute to $\mathrm{AD}$ diagnosis in patients.

Furthermore, we checked if chromosomal loss could be related to age, diagnosis time and cognitive function of AD patients. However, we did not find evidence for that. Still, chromosome loss, even in low level, might lead to phenotypic changes [31]. Genes that have been implicated in $\mathrm{AD}$ development are located in chromosomes that were lost in $\mathrm{AD}$ patients analyzed here. The genes APP, (chromosome 21q21.3), PSEN-1 (chromosome 14q24.2) and APOE (chromosome 19q13.2) encode major proteins involved in the beta-amyloid pathway. APP is the precursor of the primary component of the beta-amyloid plaques; PSEN-1 plays a role in the proteolytic cleavage of APP; and APOE is involved in the clearance of beta-amyloid from the $\mathrm{AD}$ brain $[2,3]$. Also, the gene MAPT (chromosome 17q21.1) encodes a Tau protein that is the main component of the neurofibrillary tangles in its hyperphosphorylated form. Moreover, risk genes identified recently by genome-wide analysis are also found in such chromosomes. These genes have a function in cellular signaling (CD2AP, chromosome 6p12.3), innate immunity (EPHA1, chromosome 7q34; and CLU, chromosome 8p21.1) and lipid metabolism (ABCA7, chromosome 19p13.3) [3-4]. Given that most AD patients analyzed in this study had severe cognitive impairment, systemic aneuploidy might be contributing to the disease pathology.

\section{Conclusions}

In summary, our karyotypic analyses of lymphocytes of patients from Manaus/Amazonas revealed the occurrence of a high frequency of aneuploidies in $\mathrm{AD}$ patients. Also, rare events of double and multiple aneuploidies are being reported in association with $\mathrm{AD}$ for the first time. Our results corroborate that the increase in the frequencies of aneuploidies is not related to the aging process itself, but that it is part of the disease development. However, we did not find any association between chromosomal losses and age, diagnosis time or MMSE scores. Also, no chromosomes were preferentially affected in all AD patients, and, thus, no consistent karyotype pattern for AD lymphocytes was found. Therefore, our results do 
not support the use of standard cytogenetics as a tool for AD diagnosis. Further studies with more patients and other cytogenetics tools are necessary to understand better the association between chromosomal alterations and AD.

\section{References}

[1] Morley, J. E., Farr, S. A., and Nguyen, A. D. 2018. “Alzheimer Disease.” Clinics in Geriatric Medicine 34: 591-601.

[2] Barber, R. C. 2012. "The Genetics of Alzheimer’s Disease.” Hindawi Publishing Corporation Scientific 2012. doi: $10.6064 / 2012 / 246210$.

[3] Tanzi, R. E. 2012. "The Genetics of Alzheimer Disease.” Cold Spring Harbor Perspectives in Medicine 2 (10): a006296.

[4] Bettens, K., Sleegers, K., and Broeckhoven, C. V. 2013. "Genetic Insights in Alzheimer's Disease." The Lancet Neurology 12: 92-104.

[5] Bekris, L. M., Yu, C. E., Bird, T. D., and Tsuang, D. W. 2010. “Genetics of Alzheimer Disease.” Journal of Geriatric Psychiatry and Neurology 23 (4): 213-27.

[6] Sochocka, M., Zwolińska, K., and Leszek, J. 2017. “The Infectious Etiology of Alzheimer's Disease.” Current Neuropharmacology 15 (7): 996-1009.

[7] Tasaki, S., Gaiteri, C., Mostafavi, S., De Jager, P. L., and Bennett, D. A. 2018. "The Molecular and Neuropathological Consequences of Genetic Risk for Alzheimer's Dementia.” Frontiers in Neuroscience 12: 1-15.

[8] White, B. J., Crandall, C., Goudsmit, J., Morrow, C. H., Alling, D. W., Gajdusek, D. C., et al. 1981. "Cytogenetic Studies of Familial and Sporadic Alzheimer Disease.” American Journal of Medical Genetics Part B: Neuropsychiatric Genetic 10: 77-89.

[9] Buckton, K. E., Whalley, L. J., Lee, M., and Christie, J. E. 1983. "Chromosome Changes in Alzheimer's Presenile Dementia.” Journal of Medical Genetics 20: 46-51.

[10] Migliore, L., Testa, A., Scarpato, R., Pavese, N., Petrozzi, L., and Bonuccelli, U. 1997. "Spontaneous and Induced Aneuploidy in Peripheral Blood Lymphocytes of Patients with Alzheimer's Disease.” Human Genetics 101 (3): 299-305.

[11] Poduslo, S. E., and Yin, X. 2001. "Chromosome 12 and Late-Onset Alzheimer's Disease.” Neuroscience Letters 310 (2-3): 188-90.

[12] Arendt, T. 2012. "Cell Cycle Activation and Aneuploid Neurons in Alzheimer's Disease.” Molecular Neurobiology 46: 125-35.

[13] Zheng, X., Demirci, F. Y., Barmada, M. M., Richardson,
G. A., Lopez, O. L., Sweet, R. A., Kamboh, M. I., and Feingold, E. 2014. "A Rare Duplication on Chromosome 16p11.2 Is Identified in Patients with Psychosis in Alzheimer's Disease.” PLoS One 9 (11): e111462.

[14] Yurov, Y. B., Vorsanova, S. G., Liehr, T., Kolotii, A. D., and Iourov, I. Y. 2014. "X Chromosome Aneuploidy in the Alzheimer's Disease Brain.” Molecular Cytogenetics 7: 20.

[15] Potter, H., Granic, A., and Caneus, J. 2016. "Role of Trisomy 21 Mosaicism in Sporadic and Familial Alzheimer's Disease.” Current Alzheimer Research 13 (1): 7-17.

[16] Iourov, I. Y., Vorsanova, S. G., Liehr, T., and Yurov, Y. B. 2009. "Aneuploidy in the Normal, Alzheimer's Disease and Ataxia-Telangiectasia Brain: Differential Expression and Pathological Meaning." Neurobiology of Disease 34: 212-20.

[17] Iourov, I. Y., Vorsanova, S. G., and Yurov, Y. B. 2011. "Genomic Landscape of the Alzheimer's Disease Brain: Chromosome Instability-Aneuploidy, but Not Tetraploidy-Mediates Neurodegeneration.” Neurobiology of Disease 8 (1-2): 35-7.

[18] Potter, H. 1991. "Review and Hypothesis: Alzheimer Disease and Down Syndrome-Chromosome 21 Nondisjunction May Underlie Both Disorders.” American Journal of Human Genetics 48: 1192-200.

[19] Spremo-Potparevic, B., Zivkovic, L., Djelic, N., and Bajic, V. 2004. “Analysis of Premature Centromere Division (PCD) of the $\mathrm{X}$ Chromosome in Alzheimer Patients through the Cell Cycle.” Experimental Gerontology 39 (5): 849-54.

[20] Folstein, M. F., Folstein, S. E., and Mchugh, P. R. 1975. “"Mini-mental State': A Practical Method for Grading the Cognitive State of Patients for the Clinician.” Journal of Psychiatric Research 12: 189-98.

[21] Tombaugh, T. N., and McIntyre, N. J. 1992. “The Mini-mental State Examination: A Comprehensive Review." Journal of the American Geriatrics Society 40 (9): 922-35.

[22] Moorhead, P. S., Nowell, P. C., Mellman, W. J., Battips, D. M., and Hungerford, D. A. 1960. "Chromosome Preparations of Leukocytes Cultured from Human Peripheral Blood.” Experimental Cell Research 20: 613-6.

[23] Gold, J. R., Li, Y. C., Shipley, N. S., and Powers, P. K. 1990. "Improved Methods for Working with Fish Chromosomes with a Review of Metaphase Chromosome Banding.” Journal of Fish Biology 37: 563-75.

[24] Espinosa, A., Hernández-Olasagarre, B., Moreno-Grau, S., Kleineidam, L., Heilmann-Heimbach, S., Hernández, I., et al. 2018. "Exploring Genetic Associations of Alzheimer's Disease Loci with Mild Cognitive Impairment Neurocognitive Endophenotypes." Frontiers in Aging Neuroscience 10: 340. 
[25] Migliore, L., Botto, N., Scarpato, R., Petrozzi, L., Cipriani, G., and Bonuccelli, U. 1999. "Preferential Occurrence of Chromosome 21 Malsegregation in Peripheral Blood Lymphocytes of Alzheimer Disease Patients.” Cytogenetics and Cell Genetics 87: 41-6.

[26] Cook, R. H., Ward, B. E, and Austin, J. H. 1979. "Studies in Aging of the Brain. IV. Familial Alzheimer Disease: Relation to Transmissible Dementia, Aneuploidy, and Microtubular Defects.” Neurology 29 (10): 1402-12.

[27] Ward, B. E., Cook, R. H., Robinson, A., Austin, J. H., and Summitt, R. L. 1979. "Increased Aneuploidy in Alzheimer Disease.” American Journal of Medical Genetics 3 (2): 137-44.

[28] Geller, L. N., and Potter, H. 1999. "Chromosome Missegregation and Trisomy 21 Mosaicism in Alzheimer's Disease.” Neurobiology of Disease 6 (3): 167-79.

[29] Thomas, P., and Fenech, M. 2007. "Chromosome 17 and 21 Aneuploidy in Buccal Cells Is Increased with Ageing and in Alzheimer's Disease.” Mutagenesis 23 (1): 57-65.

[30] Potter, H. 2008. "Down's Syndrome and Alzheimer's Disease: Two Sides of the Same Coin.” Future Neurology 3 (1): 29-37.

[31] Zekanowski, C., and Wojda, U. 2009. “Aneuploidy,
Chromosomal Missegregation, and Cell Cycle Reentry in Alzheimer's Disease.” Acta Neurobiologiae Experimentalis 69 (2): 232-53.

[32] Thomas, P., and Fenech, M. 2007. "A Review of Genome Mutation and Alzheimer's Disease.” Mutagenesis 22 (1): 15-33.

[33] Kormann-Bortolotto, M. H., Smith, M. D. A. C., and Neto, J. T. 1993. “Alzheimer's Disease and Ageing: A Chromosomal Approach.” Gerontology 39 (1): 1-6.

[34] Bajić, V. P., Spremo-Potparević, B., Živković, L., Bonda, D. J., Siedlak, S. L., and Casadesus, G. 2009. "The $\mathrm{X}$-chromosome Instability Phenotype in Alzheimer's Disease: A Clinical Sign of Accelerating Aging?” Medical Hypotheses 73: 917-20.

[35] Karaman, A., and Kabalar, E. 2008. "Double Aneuploidy in a Turkish Child: Down-Klinefelter Syndrome." Congenital Anomalies 48 (1): 45-7.

[36] Lee, M. N., Choi, K. H., Kim, D. K., and Kim, S. H. 2014. "Mosaic Double Aneuploidy (45, X/47, XX, +8) with Aortic Dissection.” Genetic Counseling 25 (2): 177-82.

[37] Subramaniyam, S., Mathew, S., and Pulijaal, V. 2014. "Double and Multiple Chromosomal Aneuploidies in Spontaneous Abortions: A Single Institutional Experience.” Journal of Human Reproductive Sciences 7 (4): 262-8. 